\title{
Boundary conditions for polarized radiative transfer with incident radiation
}

\author{
M. Faurobert ${ }^{1}$, I. Milici ${ }^{1,2}$, and O. Atanacković ${ }^{3}$ \\ 1 UMR 7293 Lagrange, Université de Nice Sophia Antipolis, CNRS, Observatoire de la Côte d'Azur, Campus Valrose, 06108 Nice, \\ France \\ e-mail: marianne. faurobert@unice.fr \\ 2 Astronomical observatory Belgrade, Volgina 7, 11060 Belgrade, Serbia \\ 3 Department of Astronomy, Faculty of Mathematics, University of Belgrade, Studentski Trg 16, 11000 Belgrade, Serbia
}

Received 17 July 2013 / Accepted 18 September 2013

ABSTRACT

\begin{abstract}
Context. Polarized radiative transfer in the presence of scattering in spectral lines and/or in continua may be cast in a reduced form for six reduced components of the radiation field. In this formalism, the six components of the reduced source function are angleindependent quantities. It thus drastically reduces the storage requirement of numerical codes and it is very well suited to solving polarized non-local thermodynamic equilibrium radiative transfer problems in 3D media.

Aims. This approach encounters a fundamental problem when the medium is illuminated by a polarized incident radiation, because there is a priori no way of relating the known (and measurable) Stokes parameters of the incident radiation to boundary conditions for the reduced equations. The origin of this problem is that there is no unique way of deriving the radiation-reduced components from its Stokes parameters (only the inverse operation is clearly defined). The method proposed here aims at enabling to work with arbitrary incident radiation field (polarized or unpolarized).

Methods. In previous studies, an ad-hoc treatment of the boundary conditions, applied to cases where the incident radiation is unpolarized, has been used. In this paper, we show that it is possible to account for the incident radiation in a rigorous way without any assumption on its properties by expressing the radiation field as the sum of a directly transmitted radiation and of a diffuse radiation. This approach was first used by Chandrasekhar to solve the problem of diffuse reflection by planetary atmospheres illuminated by their host star.

Results. The diffuse radiation field obeys a transfer equation with no incident radiation that may be solved in the reduced form. The first scattering of the incident radiation introduces primary creation terms in the six components of the reduced source function. Once the reduced polarized transfer problem is solved for the diffuse radiation field, its Stokes parameters can be computed. The full radiation field is then obtained by adding the directly transmitted radiation field computed in the Stokes formalism.

Conclusions. In the case of an unpolarized incident radiation, the diffuse field approach allows us to validate the previously introduced ad-hoc expressions. The diffuse field approach however leads to more accurate computation of the source terms in the case where the incident radiation is anisotropic. It is the only possible approach when the incident radiation field is polarized. We perform numerical computations of test cases, showing that the emergent line-polarization may be significantly affected by the polarization of the incident radiation.
\end{abstract}

Key words. polarization - radiative transfer - scattering - methods: analytical

\section{Introduction}

Solving non-local thermodynamic equilibrium (NLTE) polarized radiative transfer problems in multidimensional media is a challenge from a numerical point of view. In principle, it requires much more memory and computing time than the unpolarized problem, because the source function for polarized radiation depends on both the spatial coordinates and the two angles $(\theta, \varphi)$, which define the propagation direction of the radiation. Furthermore, a polarized radiation is described by four measurable quantities, the Stokes parameters (instead of one, in the unpolarized scalar case), which are coupled by the physics of the absorption and emission processes.

In this paper, we limit ourselves to problems where the polarization arises from scattering processes and where the absorption of radiation does not introduce any coupling between the Stokes parameters. This is the case for line scattering problems when the Zeeman sublevels of the lower-level are evenly populated, and for Rayleigh or Thomson scattering problems in continua. We consider only linearly polarized radiation, as scattering processes do not couple linear and circular polarization. The
Hanle effect due to the presence of weak magnetic fields is accounted for in the line scattering case.

The common point of these problems from a mathematical point of view is that the scattering can be described by a scattering matrix, which depends on both the directions and frequencies of the incoming and outgoing photons. It also depends on the magnetic field strength and direction in the presence of Hanle effect. A significant step toward the efficient solution of scattering problems in 3D media has been achieved thanks to the work of Anusha \& Nagendra (2011a,b) and the subsequent series of papers by the same authors. Their work is based on the previous paper of Frisch (2007), who showed that the scattering phase matrix can be written as the sum of terms where the ingoing and outgoing directions are factorized. This factorization applies when the frequency redistribution due to scattering is independent of the directions of the photons. This is the case when the line is formed with complete frequency redistribution, whereas it relies on the assumption that the angle-averaged frequency redistribution function may be used instead of the full angledependent one in the presence of partial frequency redistribution. In this paper, we work in the framework of this assumption, but 
it could be relaxed following the same approach as in Supriya et al. (2013).

The NLTE polarized radiative transfer equation for the three coupled Stokes parameters $I, Q$, and $U$ requires the computation of three source terms, depending on the position, the frequency, and two angles that define the radiation propagation direction. We see in the following that the reduced formalism allows us to deal with six reduced source terms, which do not depend on the propagation direction. In multidimensional media, the radiation field may be locally highly anisotropic and the anisotropy is an important source of polarization; fine angular grids are thus required for accurate numerical solutions of the polarized radiative transfer equation. The reduced formalism then drastically reduces the memory and computing time requirements of numerical solutions.

\section{Reduced formalism for polarized radiative transfer}

Let us summarize the main steps of the derivation leading to the reduced polarized radiative transfer equation.

\subsection{Reduced radiative transfer equation}

We assume that the scattering phase matrix may be written as a sum of factorized terms where the frequency and angular variables are separated,

$$
\begin{aligned}
\hat{\mathbf{R}}\left(x, \Omega, x^{\prime}, \Omega^{\prime}, \boldsymbol{B}\right)= & {\left[r_{\mathrm{II}}\left(x, x^{\prime}\right) \hat{\mathbf{P}}_{\mathrm{II}}\left(\Omega, \Omega^{\prime}, \boldsymbol{B}\right)\right.} \\
& \left.+r_{\mathrm{III}}\left(x, x^{\prime}\right) \hat{\mathbf{P}}_{\mathrm{III}}\left(\Omega, \Omega^{\prime}, \boldsymbol{B}\right)\right],
\end{aligned}
$$

where $r_{\mathrm{II}}\left(x, x^{\prime}\right)$ and $r_{\mathrm{III}}\left(x, x^{\prime}\right)$ are the standard angle-averaged redistribution functions that correspond to coherent scattering and to complete frequency redistribution in the atomic rest frame, respectively. The matrices $\hat{\mathbf{P}}_{\mathrm{II}}$ and $\hat{\mathbf{P}}_{\mathrm{III}}$ are given in the Appendix C of Anusha \& Nagendra (2011b), according to the results established in Bommier (1997a,b). They are related to the Rayleigh and Hanle phase matrices, which may be further factorized in terms of the irreducible spherical tensors $\mathcal{T}_{Q}^{K}(i, \Omega)$ as first introduced in Landi Degl'Innocenti (1984) (see also Landi Degl'Innocenti \& Landolfi 2004).

The Hanle phase matrix is given by

$$
\begin{aligned}
P_{\mathrm{i}, \mathrm{j}}^{\mathrm{H}}\left(\Omega, \Omega^{\prime}, \boldsymbol{B}\right)= & \sum_{K, Q} \mathcal{T}_{Q}^{K}(i, \Omega) \\
& \times \sum_{Q^{\prime}} \mathcal{M}_{Q Q^{\prime}}^{K}(\boldsymbol{B})(-1)^{Q^{\prime}} \mathcal{T}_{-Q^{\prime}}^{K}\left(j, \Omega^{\prime}\right),
\end{aligned}
$$

where $\Omega$ and $\Omega^{\prime}$ denote the propagation directions of the outgoing and ingoing photons, respectively, and $x$ and $x^{\prime}$ are their frequencies measured with respect to the line central frequency in units of Doppler width, and $\boldsymbol{B}$ is the magnetic field vector. The index $i=0,1,2,3$ refers to the four Stokes parameters. The matrix $\mathcal{M}_{Q, Q^{\prime}}^{K}(B)$ is given in Landi Degl'Innocenti \& Landolfi (2004).

In the absence of a magnetic field, the Rayleigh phase matrix reduces to

$P_{\mathrm{i}, \mathrm{j}}^{\mathrm{R}}\left(\Omega, \Omega^{\prime}\right)=\sum_{K, Q} \mathcal{T}_{Q}^{K}(i, \Omega)(-1)^{Q} \mathcal{T}_{-Q}^{K}\left(j, \Omega^{\prime}\right)$

The irreducible spherical tensors are well suited to handling the angular dependence of Rayleigh-type scattering phase matrices with $K=0,1,2$ and $-K \leq Q \leq K$. In these problems, the circular and linear polarization decouple.
Using these factorizations, Anusha \& Nagendra (2011a,b) showed that the polarized radiative transfer equation along the ray for the Stokes vector $\boldsymbol{I}=(I, Q, U)^{\mathrm{T}}$, namely

$\frac{\mathrm{d} \boldsymbol{I}(\boldsymbol{r}, \Omega, x)}{\mathrm{d} s}=-k_{\mathrm{tot}}(\boldsymbol{r}, x)[\boldsymbol{I}(\boldsymbol{r}, \Omega, x)-\boldsymbol{S}(\boldsymbol{r}, \Omega, x)]$,

may be cast into a set of six formally independent transfer equations for the six-component reduced Stokes vector $\mathcal{I}=$ $\left(I_{0}^{0}, I_{0}^{2}, I_{-1}^{2}, I_{1}^{2}, I_{-2}^{2}, I_{2}^{2}\right)^{\mathrm{T}}$, where the corresponding six-component reduced source vector $\mathcal{S}(\boldsymbol{r}, x)$ is angle-independent. That is

$\frac{\mathrm{d} \mathcal{I}(\boldsymbol{r}, \Omega, x)}{\mathrm{d} s}=-k_{\mathrm{tot}}(\boldsymbol{r}, x)[\mathcal{I}(\boldsymbol{r}, \Omega, x)-\mathcal{S}(\boldsymbol{r}, x)]$.

As usual, $k_{\mathrm{tot}}(\boldsymbol{r}, x)=k_{\mathrm{l}}(\boldsymbol{r}) \phi(x)+k_{\mathrm{c}}(\boldsymbol{r})$, where $k_{\mathrm{l}}$ and $k_{\mathrm{c}}$ denote, respectively, the line-integrated and the continuum absorption coefficients, and $\phi(x)$ is the normalized line absorption profile. The coordinate $s$ is the path length along the ray.

The reason for this remarkable property is that the source vector in Eq. (4) may be written in the irreducible spherical tensors basis as

$S_{i}(\boldsymbol{r}, \Omega, x)=\sum_{Q K} \mathcal{T}_{Q}^{K}(i, \Omega) S_{Q}^{K}(\boldsymbol{r}, x)$.

Here the irreducible components $S_{Q}^{K}(\boldsymbol{r}, x)$ of the total source vector are independent of the propagation direction. This is a direct consequence of the factorization of the scattering phase matrix.

As already mentioned in Frisch (2007), the reduced intensity components defined as the solutions of the reduced radiative transfer equations are different from the tensors $\mathcal{I}_{Q}^{K}$, defined in Landi Degl'Innocenti \& Landolfi (2004), whose angle-averages appear in the statistical equilibrium equations for the element of the atomic density matrix.

The $S_{Q}^{K}$ components of the total source function are simply related to the reduced components of the line and continuum source functions $S_{1, Q}^{K}$ and $S_{\mathrm{c}, Q}^{K}$ by

$S_{Q}^{K}(\boldsymbol{r}, x)=\frac{k_{\mathrm{l}}(\boldsymbol{r}) \phi(x)}{k_{\mathrm{tot}}} S_{1, Q}^{K}(\boldsymbol{r}, x)+\frac{k_{\mathrm{c}}(\boldsymbol{r})}{k_{\mathrm{tot}}} S_{\mathrm{c}, Q}^{K}(\boldsymbol{r}, x)$.

The reduced line source function is given in Eqs. (18)-(19) of Anusha \& Nagendra (2011b) in terms of the reduced sixcomponent radiation vector $\mathcal{I}$, so we may write it as

$\mathcal{S}_{1}(\boldsymbol{r}, x)=\int_{-\infty}^{\infty} \mathrm{d} x^{\prime} \int \frac{\mathrm{d} \Omega^{\prime}}{4 \pi} \frac{\hat{\mathbf{R}}\left(x, x^{\prime}, \boldsymbol{B}\right)}{\phi(x)} \hat{\mathbf{\Psi}}\left(\Omega^{\prime}\right) \mathcal{I}\left(\boldsymbol{r}, \Omega^{\prime}, x^{\prime}\right)+\mathcal{G}_{1}(\boldsymbol{r})$,

where the $6 \times 6$ matrix $\hat{\mathbf{\Psi}}$ is given by

$\hat{\mathbf{\Psi}}_{Q, Q^{\prime}}^{K, K^{\prime}}(\Omega)=\sum_{j=0}^{3}(-1)^{Q} \mathcal{T}_{-Q}^{K}(j, \Omega) \mathcal{T}_{Q^{\prime}}^{K^{\prime}}(j, \Omega)$.

In Eq. (8), the second term of the right-hand side denotes the reduced components of the primary source of line photons, and $\hat{\mathbf{R}}\left(x, x^{\prime}, \boldsymbol{B}\right)$ is a generic notation for a $(6 \times 6)$ angle-averaged frequency redistribution matrix. Its analytical expressions, which vary according to different frequency domains, can be derived from the formulae given in Appendices B and C of Anusha \& Nagendra (2011b).

The reduced continuum source function may be cast into a form similar to Eq. (8), replacing $G_{1}(\boldsymbol{r})$ by the primary source of 
photons in the continuum $\mathcal{G}_{\mathrm{c}}(\boldsymbol{r})$ and the frequency redistribution matrix $\hat{\mathbf{R}}\left(x, x^{\prime}, \boldsymbol{B}\right)$ by $\delta\left(x-x^{\prime}\right) \hat{\mathbf{1}}$ where $\hat{\mathbf{1}}$ is the identity matrix.

The reduced source function $\mathcal{S}$ and intensity vector $\mathcal{I}$ are complex quantities, to deal with real quantities Frisch (2007) introduced the following notations:

$I_{Q}^{K, x}(\boldsymbol{r}, \Omega, x)=\operatorname{Re}\left(I_{Q}^{K}(\boldsymbol{r}, \Omega, x)\right)$,

$I_{Q}^{K, y}(\boldsymbol{r}, \Omega, x)=\operatorname{Im}\left(I_{Q}^{K}(\boldsymbol{r}, \Omega, x)\right)$.

She showed that $\mathcal{I}^{r}=\left(I_{0}^{0}, I_{0}^{2}, I_{1}^{2, x}, I_{1}^{2, y}, I_{2}^{2, x}, I_{2}^{2, y}\right)^{\mathrm{T}}$ obeys a radiative transfer equation analogous to Eq. (5), where the corresponding source term $\mathcal{S}^{r}$ can be derived from expressions analogous to Eq. (8) when the matrix $\hat{\boldsymbol{\Psi}}$ is replaced by the matrix $\hat{\mathbf{\Psi}}^{r}$, as given in Appendix D of Anusha \& Nagendra (2011b). The derivation of the transfer equation for the real reduced components of the radiation field relies on the symmetry property $\left(I_{Q}^{K}\right)^{*}=(-1)^{Q} I_{-Q}^{K}$.

The reduced formalism for polarized transfer that we have summarized here is well suited to an efficient numerical solution, such as Polarized Accelerated Lambda Iterations (PALI) or Jacobi methods, which may be applied in 3D media. Once the reduced components of the radiation field have been obtained, one can easily compute the Stokes parameters of the radiation field using the relations given in the Appendix B of Frisch (2007).

\subsection{Boundary conditions for the reduced radiative transfer equation}

In the papers quoted above, the radiative transfer problem was solved for self-emitting media in the absence of incident radiation. In Milić (2013), the same formalism was used but for computing line-scattering polarization emerging from a circumstellar disk illuminated by its central star. To self-consistently solve the reduced transfer equation, one needs to cast the boundary conditions, i.e. the known incident radiation field, into the same reduced basis. However, there is no unique way of deriving the reduced components $I_{Q}^{K}$ from a known radiation field described in terms of the Stokes parameters (only the reversed operation is well defined). The reason is probably that the reduced formalism is well suited to describe the angular dependence of a radiation field that arises from scattering processes, or from unpolarized and isotropic primary creation of photons, but it is not clear how to apply it to any arbitrary radiation. For problems where the medium is illuminated by an arbitrary radiation field, which can be polarized or unpolarized, we have to find a way of writing the boundary conditions for Eq. (5).

In the following, we show that a consistent way of solving the problem is to write the radiation field as the sum of a transmitted radiation and a diffuse part. The diffuse radiation obeys a transfer equation like Eq. (5) with an additional primary creation term that arises from the first scattering of the incident radiation in the medium. By definition, the diffuse radiation transfer problem has no incident radiation. The additional primary source term is easily cast into reduced components because it originates in a scattering process. The diffuse radiation may then be computed in the reduced formalism presented above and its Stokes parameters can then be derived. The full radiation is finally obtained in the Stokes formalism by adding the Stokes components of the transmitted radiation and the Stokes components of the diffuse radiation.

\section{Transmitted and diffuse radiation fields}

We now deal with the polarized radiative transfer equation in the Stokes formalism, given by Eq. (4). Here we want to solve it the case where the medium is illuminated by an incident radiation field denoted by $\boldsymbol{I}_{\text {inc }}\left(\boldsymbol{r}_{0}, \Omega, x\right)$ with known Stokes parameters $I_{\text {inc }}^{i}\left(\boldsymbol{r}_{0}, \Omega, x\right)$, where $i=0,1,2,3$. As in Chandrasekhar (1960) and Ivanov et al. (1997), we write the solution of Eq. (4) as the sum of two terms:

$$
\boldsymbol{I}(\boldsymbol{r}, \Omega, x)=\boldsymbol{I}_{\text {inc }}\left(\boldsymbol{r}_{0}, \Omega, x\right) \exp \left(-\tau_{\Omega, x}(\boldsymbol{r})\right)+\boldsymbol{I}_{\mathrm{d}}(\boldsymbol{r}, \Omega, x),
$$

where $\tau_{\Omega, x}(\boldsymbol{r})$ is the line optical depth from the boundary at the location $\boldsymbol{r}$ along the direction $\Omega$ at frequency $x$. The first term in the right-hand side of Eq. (11) is the transmitted radiation at depth $\boldsymbol{r}$ and the second term is the diffuse radiation field. Substituting Eq. (11) in Eq. (4), we easily derive the radiative transfer equation for $\boldsymbol{I}_{\mathrm{d}}(\boldsymbol{r}, \Omega, x)$, namely

$$
\frac{\mathrm{d} \boldsymbol{I}_{\mathrm{d}}(\boldsymbol{r}, \Omega, x)}{\mathrm{d} s}=-k_{\mathrm{tot}}(\boldsymbol{r}, x)\left[\boldsymbol{I}_{\mathrm{d}}(\boldsymbol{r}, \Omega, x)-\boldsymbol{S}(\boldsymbol{r}, \Omega, x)\right] .
$$

The source term is the same as in Eq. (4); it may be written in terms of the diffuse and transmitted radiation by substituting Eq. (11) into the scattering integral. The scattering integral is now the sum of two terms, of which both may be expanded on the irreducible tensors $\mathcal{T}_{Q}^{K}(i, \Omega)$. Thus, we can repeat for the diffuse radiation field the same procedure as described in the previous section and cast the radiative transfer Eq. (12) into a reduced form similar to Eq. (5), where the reduced source vector is given in Eq. (8).

Here, we write the line source vector $\mathcal{S}_{1}^{r}$ on its real form in terms of the diffuse field $\mathcal{I}_{\mathrm{d}}^{r}$. Namely,

$$
\begin{aligned}
\mathcal{S}_{1}^{r}(\boldsymbol{r}, x)= & \int_{-\infty}^{\infty} \mathrm{d} x^{\prime} \int \frac{\mathrm{d} \Omega^{\prime}}{4 \pi} \frac{\hat{\mathbf{R}}\left(x, x^{\prime}, \boldsymbol{B}\right)}{\phi(x)} \hat{\mathbf{\Psi}}^{r}\left(\Omega^{\prime}\right) \mathcal{I}_{\mathrm{d}}^{r}\left(\boldsymbol{r}, \Omega^{\prime}, x^{\prime}\right) \\
& +\mathcal{G}_{1}(\boldsymbol{r})+C^{r}(\boldsymbol{r}, x),
\end{aligned}
$$

where $C^{r}=\left(C_{0}^{0}, C_{0}^{2}, C_{1}^{2, x}, C_{1}^{2, y}, C_{2}^{2, x}, C_{2}^{2, y}\right)^{\mathrm{T}}$ is the six-component additional source term, related to the real and imaginary parts of the complex quantities,

$$
\begin{aligned}
C_{Q}^{K}(\boldsymbol{r}, x)= & \frac{W_{K}}{\phi(x)} \int_{-\infty}^{\infty} \mathrm{d} x^{\prime} \int \frac{\mathrm{d} \Omega^{\prime}}{4 \pi} \mathrm{e}^{-\tau\left(\Omega^{\prime}, x^{\prime}\right)} \\
& \times \sum_{j=0}^{3} \mathbf{R}_{Q, j}^{K}\left(x, x^{\prime}, \Omega^{\prime}, \boldsymbol{B}\right) I_{\mathrm{inc}}^{j}\left(\boldsymbol{r}_{0}, \Omega^{\prime}, x^{\prime}\right) .
\end{aligned}
$$

The coefficients $W_{K}$ are constants that depend on the quantum numbers of the line atomic levels. Analytical expressions of $C^{r}$ as a function of the Stokes parameters of the incident radiation are given in Appendix A. The continuum source function in the reduced form has an expression similar to Eq. (13) but for coherent Rayleigh scattering.

In non-magnetic media, the redistribution matrix in Eq. (14) reduces to

$\mathbf{R}_{Q, j}^{K}\left(x, x^{\prime}, \Omega^{\prime}\right)=r^{K}\left(x, x^{\prime}\right)(-1)^{Q} \mathcal{T}_{-Q}^{K}\left(j, \Omega^{\prime}\right)$,

with

$$
\begin{aligned}
r^{K}\left(x, x^{\prime}\right)= & \frac{\Gamma_{R}}{\Gamma_{R}+\Gamma_{I}+\Gamma_{E}}\left[r_{\mathrm{II}}\left(x, x^{\prime}\right)\right. \\
& \left.+r_{\mathrm{III}}\left(x, x^{\prime}\right) \times \frac{\Gamma_{E}-D^{(K)}}{\Gamma_{R}+\Gamma_{I}+D^{(K)}}\right] .
\end{aligned}
$$

In the presence of Hanle effect, we recall that the redistribution matrix $\mathbf{R}_{Q, j}^{K}$ has different expressions, according to different frequency domains (see Appendix B in 
Anusha \& Nagendra 2011b). For the lines formed in dilute media (such as prominences) where collisions are negligible, we have:

$$
\begin{aligned}
\mathbf{R}_{Q, j}^{K}\left(x, x^{\prime}, \Omega^{\prime}, \boldsymbol{B}\right)= & \sum_{Q^{\prime}} \mathcal{M}_{Q, Q^{\prime}}^{K}(B)(-1)^{Q^{\prime}} \mathcal{T}_{-Q^{\prime}}^{K}\left(j, \Omega^{\prime}\right) \\
& \times r_{\mathrm{II}}\left(x, x^{\prime}\right),
\end{aligned}
$$

in the frequency domain where $x\left(x+x^{\prime}\right)<2 v_{\mathrm{c}}^{2}(a)$ and $x^{\prime}\left(x+x^{\prime}\right)<$ $2 v_{\mathrm{c}}^{2}(a)$. In the complementary domain,

$\mathbf{R}_{Q, j}^{K}\left(x, x^{\prime}, \Omega^{\prime}, \boldsymbol{B}\right)=(-1)^{Q} \mathcal{T}_{-Q}^{K}\left(j, \Omega^{\prime}\right) r_{\mathrm{II}}\left(x, x^{\prime}\right)$.

The cutoff frequency $v_{\mathrm{c}}$ between the line core and line wings depends on the value of the Voigt parameter $a$. It is usually on the order of a few Doppler widths.

The diffuse radiation field is zero at the boundaries of the medium. We can then solve its radiative transfer equation in reduced form and derive its Stokes parameters from the relations given in Appendix B of Frisch (2007). When the Stokes parameters of the diffuse field are computed, we obtain the Stokes parameters of the total radiation field through Eq. (11).

\section{Numerical tests}

The numerical solution of the reduced transfer problem for the diffuse radiation may be performed with the Jacobi iterative method introduced in Anusha \& Nagendra (2011a; see also Milić 2013). The additional source terms $C^{r}(\boldsymbol{r}, x)$ and $C_{\mathrm{c}}^{r}(\boldsymbol{r}, x)$ have to be computed first on long characteristics from the boundaries to each point of the spatial grid. We note that this significantly increases the computational work because of the use of the long characteristics, moreover integration must be performed on dense grids if high precision is to be achieved. For the numerical tests presented below, we use a 100-point Gaussian quadrature for integration over $\theta$, while integration over azimuth is not needed because of the symmetry of the incident radiation. For this particular test case, the use of quadrature with 20 Gaussian points results in differences smaller than $1 \%$.

In all the tests we have used a slightly modified second order short-characteristics (Olson \& Kunasz 1987) formal solution. A 2D-Forth and Back Implicit Lambda Iterative procedure (FBILI) is used to obtain a self-consistent solution of the unpolarized NLTE problem ${ }^{1}$. We then perform several $\Lambda$ iterations for the polarized part with the same formal solver to obtain the solution for the reduced source function.

\subsection{Unpolarized incident radiation field}

In Anusha \& Nagendra (2011a,b) it is assumed that one may use as boundary conditions for the reduced radiation field, if the incident radiation is not polarized, the expression $\mathcal{I}_{\text {inc }}=$ $\left(I_{\text {inc }}, 0,0,0,0,0\right)^{\mathrm{T}}$. In that case one can verify that both this approach and the diffuse radiation field approach lead to identical expressions for the Stokes parameters of the total radiation field.

However, the diffuse field approach allows us to consider the anisotropy of the incident radiation in the computation of the polarized source function in a more accurate way, even in the case when the incident radiation is unpolarized, because of the following: If one was to solve the radiative transfer problem, one would have to specify $\mathcal{I}_{\text {inc }}$ on a coarse angular grid. In the case of an anisotropic illumination, which also encounters abrupt changes (such as in the following examples), this would

\footnotetext{
1 Work in preparation by Milić \& Atanacković.
}

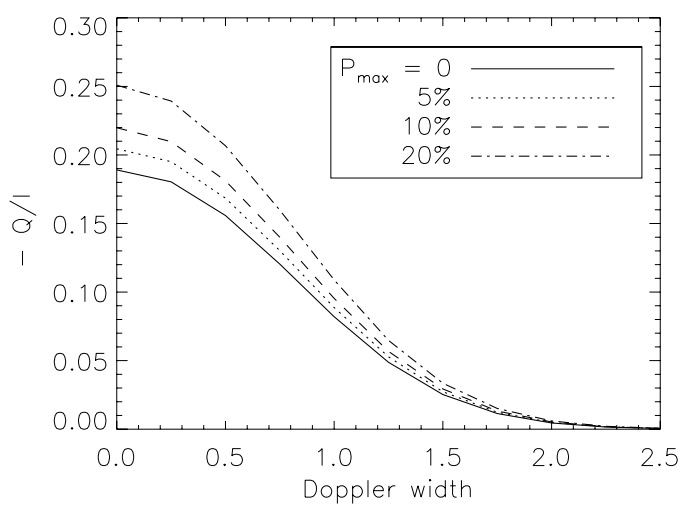

Fig. 1. $Q / I$ line profiles emergent from a vertical slab illuminated by a polarized incident radiation. The different curves correspond to different maximum degrees of polarization of the incident radiation field.

introduce errors in the computation of the reduced source vector. The accuracy is improved if one uses the averaging procedure described in Gouttebroze (2005) and subsequently in Milić (2013). However, the averaging process is constructed to accurately reproduce the $S_{0}^{0}$ component (identical to the scalar mean intensity $J$ ), while the computation of the other components is still not accurate. For externally illuminated objects of low and moderate optical thickness, the incident radiation is the dominant part of the reduced source function. This part is computed with a much higher precision with our approach because the averaging process is avoided, while the diffuse part of the computation can still be done with a coarser angular grid.

\subsection{Polarized incident radiation field}

The formalism developed in Anusha \& Nagendra (2011a) does not deal with the boundary conditions in cases where the incident radiation is polarized. In that case, we have to use the diffuse field approach. Here we consider slabs that are effectively 1D (computations are performed with a 2D code), placed horizontally or vertically above the solar surface and illuminated by a limb darkened continuum radiation given by (similar example as in: Landi Degl'Innocenti \& Landolfi 2004)

$I_{\text {inc }}=1-0.95(1-\cos \theta)+0.2(1-\cos \theta)^{2}$.

We consider a case with the following parameters: there is no continuum opacity, the line absorption profile is given by a Doppler function, the line is formed by only scattering processes and $W_{0}=W_{2}=1$ is assumed. The line-integrated optical depth in both cases is equal to unity. This creates some radiative transfer effects while still maintaining a large impact of the incident radiation. In both cases, the slab is standing $50000 \mathrm{~km}$ above the solar surface. Note that it introduces an additional geometrical anisotropy since the incident radiation is within a limited solid angle between the solar surface and the slab. As a coarse computational grid, we use a grid with $12 \times 12$ angles where integrations over $\theta$ and $\varphi$ are done with a Gaussian and trapezoid quadrature, respectively.

Figure 1 shows the emergent $Q / I$ line profiles in the direction $\theta=\pi / 2, \varphi=0$ from a vertical slab (simple model of a solar prominence), which is illuminated by a polarized radiation with the following angular dependence:

$Q=-P_{\max } \times I\left(1-\cos ^{2} \theta\right)$.

We vary $P_{\max }$ between $0 \%$ and $20 \%$. It is clearly seen that the degree of polarization of the incident radiation significantly influences the polarization in the line. A similar effect can be seen 
M. Faurobert et al.: Boundary conditions for polarized radiative transfer

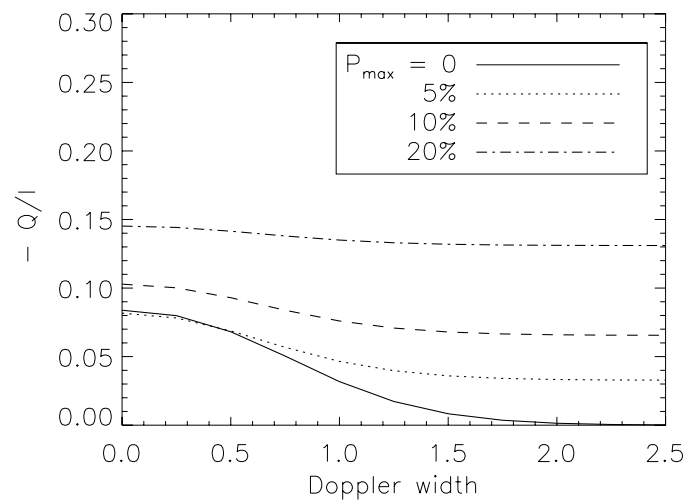

Fig. 2. Same as Fig. 1, except for a horizontal slab, $\mu=0.6$.

in Fig. 2 in the case of the horizontal slab (simple model of a solar filament). Note that the emergent radiation is a combination of the transmitted and scattered radiations in the latter case.

\section{Conclusion}

We have demonstrated a consistent way of dealing with boundary conditions for polarized radiative transfer in the framework of the reduced intensity formalism. Our method is based on splitting the radiation field into a transmitted and a diffuse component. We then incorporate the incident radiation (known in the Stokes formalism) in the source function by performing high-precision angular integration and appropriate attenuation via long characteristics. This method is computationally slower but allows for higher precision, even in the unpolarized case and, more importantly, allows accounting for polarized incident radiation.

We have applied the method on simple 1D slabs, which mimic prominences or filaments illuminated by the solar continuum radiation. We have shown that the degree of polarization of the incoming radiation may significantly influence the emergent line polarization from such structures. It is possible that the interpretation of UV lines formed by scattering of polarized continuum radiation in the upper layers of the Sun might require such computational techniques.

Acknowledgements. I.M. is thankful to Eiffel Scholarship of French Government and to COST MP1104 for supporting his stay in Nice. O.A. is thankful to the University of Nice-Sophia Antipolis for supporting her stay in Nice. This research is also partially funded by the Serbian Ministry of Science and Education through the project 176004, "Stellar Physics".

\section{Appendix A: Explicit expressions for the real reduced primary source term due to the incident radiation}

Here, we give the analytical expressions for the additional source term in the RT equation for the diffuse radiation in real form, $C^{r}=\left(C_{0}^{0}, C_{0}^{2}, C_{1}^{2, x}, C_{1}^{2, y}, C_{2}^{2, x}, C_{2}^{2, x}\right)^{\mathrm{T}}$.

In the non-magnetic case,

$$
\begin{aligned}
C_{0}^{0}(\boldsymbol{r}, x)= & \frac{W_{0}}{\phi(x)} \int_{-\infty}^{\infty} r\left(x, x^{\prime}\right) \mathrm{d} x^{\prime} \int \frac{\mathrm{d} \Omega^{\prime}}{4 \pi} I_{\mathrm{inc}}\left(\boldsymbol{r}_{0}, \Omega^{\prime}, x^{\prime}\right) \mathrm{e}^{-\tau\left(\Omega^{\prime}, x^{\prime}\right)} \\
C_{0}^{2}(\boldsymbol{r}, x)= & \frac{W_{2}}{\phi(x)} \int_{-\infty}^{\infty} r\left(x, x^{\prime}\right) \mathrm{d} x^{\prime} \int \frac{\mathrm{d} \Omega^{\prime}}{4 \pi} \mathrm{e}^{-\tau\left(\Omega^{\prime}, x^{\prime}\right)} \\
& {\left[\frac{1}{2 \sqrt{2}}\left(3 \cos ^{2} \theta-1\right) I_{\text {inc }}\left(\boldsymbol{r}_{0}, \Omega^{\prime}, x^{\prime}\right)\right.} \\
& \left.-\frac{3}{2 \sqrt{2}} \sin ^{2} \theta Q_{\text {inc }}\left(\boldsymbol{r}_{0}, \Omega^{\prime}, x^{\prime}\right)\right]
\end{aligned}
$$

$$
\begin{aligned}
& C_{1}^{2 x}(\boldsymbol{r}, x)=\frac{W_{2}}{\phi(x)} \int_{-\infty}^{\infty} r\left(x, x^{\prime}\right) \mathrm{d} x^{\prime} \int \frac{\mathrm{d} \Omega^{\prime}}{4 \pi} \mathrm{e}^{-\tau\left(\Omega^{\prime}, x^{\prime}\right)} \\
& {\left[-\frac{\sqrt{3}}{2} \sin \theta \cos \theta \cos \varphi I_{\text {inc }}\left(\boldsymbol{r}_{0}, \Omega^{\prime}, x^{\prime}\right)\right.} \\
& -\frac{\sqrt{3}}{2} \cos \theta \sin \theta \cos \varphi Q_{\text {inc }}\left(\boldsymbol{r}_{0}, \Omega^{\prime}, x^{\prime}\right) \\
& \left.+\frac{\sqrt{3}}{2} \sin \theta \sin \varphi U_{\mathrm{inc}}\left(\boldsymbol{r}_{0}, \Omega^{\prime}, x^{\prime}\right)\right] \text {, } \\
& C_{1}^{2 y}(\boldsymbol{r}, x)=\frac{W_{2}}{\phi(x)} \int_{-\infty}^{\infty} r\left(x, x^{\prime}\right) \mathrm{d} x^{\prime} \int \frac{\mathrm{d} \Omega^{\prime}}{4 \pi} \mathrm{e}^{-\tau\left(\Omega^{\prime}, x^{\prime}\right)} \\
& {\left[\frac{\sqrt{3}}{2} \sin \theta \cos \theta \sin \varphi I_{\text {inc }}\left(\boldsymbol{r}_{0}, \Omega^{\prime}, x^{\prime}\right)\right.} \\
& +\frac{\sqrt{3}}{2} \cos \theta \sin \theta \sin \varphi Q_{\text {inc }}\left(\boldsymbol{r}_{0}, \Omega^{\prime}, x^{\prime}\right) \\
& \left.+\frac{\sqrt{3}}{2} \sin \theta \cos \varphi U_{\mathrm{inc}}\left(\boldsymbol{r}_{0}, \Omega^{\prime}, x^{\prime}\right)\right] \text {, } \\
& C_{2}^{2 x}(\boldsymbol{r}, x)=\frac{W_{2}}{\phi(x)} \int_{-\infty}^{\infty} r\left(x, x^{\prime}\right) \mathrm{d} x^{\prime} \int \frac{\mathrm{d} \Omega^{\prime}}{4 \pi} \mathrm{e}^{-\tau\left(\Omega^{\prime}, x^{\prime}\right)} \\
& {\left[\frac{\sqrt{3}}{4} \sin ^{2} \theta \cos 2 \varphi I_{\text {inc }}\left(\boldsymbol{r}_{0}, \Omega^{\prime}, x^{\prime}\right)\right.} \\
& -\frac{\sqrt{3}}{4}\left(1+\cos ^{2} \theta\right) \cos 2 \varphi Q_{\text {inc }}\left(\boldsymbol{r}_{0}, \Omega^{\prime}, x^{\prime}\right) \\
& \left.+\frac{\sqrt{3}}{2} \cos \theta \sin 2 \varphi U_{\mathrm{inc}}\left(\boldsymbol{r}_{0}, \Omega^{\prime}, x^{\prime}\right)\right] \text {, } \\
& C_{2}^{2 y}(\boldsymbol{r}, x)=\frac{W_{2}}{\phi(x)} \int_{-\infty}^{\infty} r\left(x, x^{\prime}\right) \mathrm{d} x^{\prime} \int \frac{\mathrm{d} \Omega^{\prime}}{4 \pi} \mathrm{e}^{-\tau\left(\Omega^{\prime}, x^{\prime}\right)} \\
& {\left[-\frac{\sqrt{3}}{4} \sin ^{2} \theta \sin 2 \varphi I_{\text {inc }}\left(\boldsymbol{r}_{0}, \Omega^{\prime}, x^{\prime}\right)\right.} \\
& +\frac{\sqrt{3}}{4}\left(1+\cos ^{2} \theta\right) \sin 2 \varphi Q_{\text {inc }}\left(\boldsymbol{r}_{0}, \Omega^{\prime}, x^{\prime}\right) \\
& \left.+\frac{\sqrt{3}}{2} \cos \theta \cos 2 \varphi U_{\text {inc }}\left(\boldsymbol{r}_{0}, \Omega^{\prime}, x^{\prime}\right)\right] \text {. }
\end{aligned}
$$

In the presence of a magnetic field for lines formed in dilute media, the same magnetic matrix as given in Frisch (2007) can be applied to $C^{r}$ to account for the Hanle effect.

\section{References}

Anusha, L. S., \& Nagendra, K. N. 2011a, ApJ, 726, 6

Anusha, L. S., \& Nagendra, K. N. 2011b, ApJ, 738, 116

Bommier, V. 1997a, A\&A, 328, 706

Bommier, V. 1997b, A\&A, 328, 726

Chandrasekhar, S. 1960, Radiative transfer (Dover: Dover Publ.)

Frisch, H. 2007, A\&A, 476, 665

Gouttebroze, P. 2005, A\&A, 434, 1165

Ivanov, V. V., Grachev, S. I., \& Loskutov, V. M. 1997, A\&A, 321, 968

Landi Degl'Innocenti, E. 1984, Sol. Phys., 91, 1

Landi Degl'Innocenti, E., \& Landolfi, M. 2004, Polarization in Spectral Lines,

Astrophys. Space Sci. Lib. (Dordrecht: Kluwer Academic Publishers), 307

Milić, I. 2013, A\&A, 555, A130

Olson, G. L., \& Kunasz, P. B. 1987, J. Quant. Spect. Radiat. Transf., 38, 325

Supriya, H. D., Sampoorna, M., Nagendra, K. N., Ravindra, B., \& Anusha, L. S. 2013, J. Quant. Spect. Radiat. Transf., 119, 67 